\title{
Approximate analytical method for thermal analysis of an annular fin having a step profile
}

\author{
B. Kundu ${ }^{1}$, K.-S. Lee ${ }^{2}$ \& A. Campo ${ }^{3}$ \\ ${ }^{1}$ Department of Mechanical Engineering, Jadavpur University, India \\ ${ }^{2}$ School of Mechanical Engineering, Hanyang University, Korea \\ ${ }^{3}$ Department of Mechanical Engineering, \\ University of Texas at San Antonio, USA
}

\begin{abstract}
An approximate analytical technique is developed to determine the performance of a rectangular stepped annular fin with a constant base temperature. An algebraic expression based on the mean value theorem is used as an approximation tool. The corresponding temperature distribution and heat transfer characteristics in the fin are determined as exponential functions. The standard Modified Bessel function formulation exists for the exact analysis. Error analysis was carried out to compare the exact and approximate results. It is observed that the integral approach of calculating heat transfer is more accurate than the differential approach while applying the approximate analytic technique. The error associated with the approximate analytic methods is well within the accuracy level for analysis and design.

Keywords: annular fin, exact analytical method, approximate analytical method, step profile.
\end{abstract}

\section{Introduction}

Annular disk fins are attached externally to circular pipes to increase the heat transfer rate. As the cross-sectional areas of annular disc fins are constant, the fin material is not effectively used to transfer the conductive heat near the tip. Hence, different tapered profiles, for example, triangular, trapezoidal, parabolic, hyperbolic, etc., have been reported in the literature. Keeping in mind the low cost and higher heat transfer rate, designers continuously endeavor to determine optimum fin profile and shape for a specified fin volume (Kundu et al. [1]). 
To maximize the surface area to volume ratio, the fin should be thin. However, tapered profiles are difficult to manufacture. On the other hand, the fin thickness near the tip can easily be reduced in a step-wise manner by selecting a suitable step profile (Kundu and Das [2]). The profile shape of a step fin is similar to that of a constant thickness fin and, therefore, such a fin is easier to manufacture. A step fin not only properly utilizes the fin material, but also induces turbulent flow over its surface. Thus, the heat transfer coefficient for convective fins with step surfaces is higher than that of fins without step surfaces.

Kraus et al. [3] published an excellent comprehensive review of the existing literature on extended surface heat transfer. Ullmann and Kalman [4] studied the efficiency of annular fins of various tapered profiles for a constant heat transfer coefficient. Under dehumidifying surface conditions, an analysis was carried out by Kundu [5] on SRC profiles to determine fin performances. Optimization of space radiators having step fins was analyzed by Arslanturk [6]. Thermal analysis of annular fins with rectangular and hyperbolic profiles was performed in two recent publications [7, 8]. Kundu and Das [9] investigated eccentric annular disk fins, which can transfer more heat than concentric annular fins with an equal fin volume under a space restriction on one side of the circular tube.

The works of Sharqawy and Zubair [10] and Rosario and Rahman [11] deal with the performance and optimization issues when a single annular fin or an assembly of annular fins operates under dehumidifying conditions. These analyses involve simultaneous heat and mass transfers from the fin surface. To transfer heat effectively from a primary surface, T-shaped fins had been examined by Almogbel and Bejan [12] and later by Kundu and Bhanja [13-15].

In the present study, the thermal performance of an annular step fin is investigated using an approximate analytical method. The approximate method based on the mean value theorem is described as an alternative solution to avoid the rigorous calculations for the exact analysis involving the modified Bessel function. The approximate results show very good agreement with the exact results for the temperature and performance parameters.

\section{Mathematical analysis}

The analysis that is carried out revolves around an annular fin with a rectangular step profile, as shown in Fig. 1. In the fin, the inner radius is $r_{1}$, the outer radius is

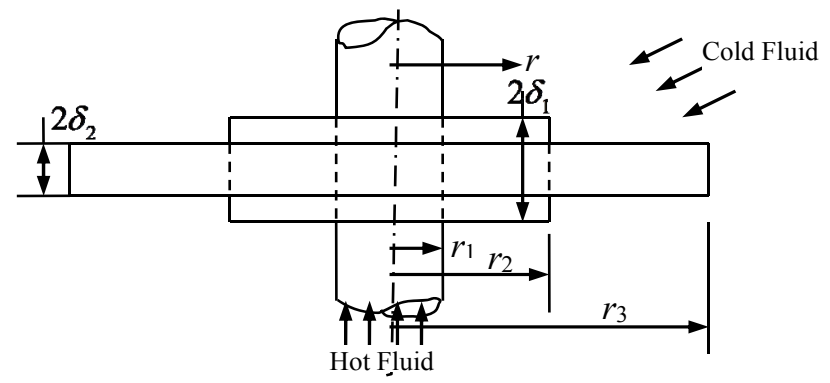

Figure 1: A view of annular step fin. 
$r_{3}$ and the step radius is $r_{2}$. The step fin is constructed by joining two different thicknesses, $2 \delta_{1}$ and $2 \delta_{2}$, at the intermediate radius $r_{1}$. The radial coordinate starts from the centre of the tube. The heat transfer coefficient over the exposed surfaces alongside the fin is $h$. The heat transfer takes place from the fin surface to the surrounding fluid due to convection only. The temperature and the dimensions can be normalized by defining

$$
\begin{array}{r}
\theta=\left(T_{1}-T_{a}\right) /\left(T_{b}-T_{a}\right) ; \phi=\left(T_{2}-T_{a}\right) /\left(T_{b}-T_{a}\right) ; \delta=\delta_{2} / \delta_{1} ; R_{1}=r_{1} / r_{3} ; \\
R_{2}=r_{2} / r_{3} ; \xi=\delta_{1} / r_{1} ; B i=h r_{1} / k .
\end{array}
$$

where, $T_{a}, T_{\mathrm{b}}, T_{1}$, and $T_{2}$ are ambient temperature, fin temperature at the base, local temperature in the radial direction for the thick part of the fin, and local temperature in the radial direction for the thin part of the fin, respectively. $k$ is the thermal conductivity of the fin material. The one-dimensional heat conduction equations for the step annular fin can be written under steady-state conditions in dimensionless form as

$$
\begin{cases}\frac{d^{2} \theta}{d R^{2}}+\frac{1}{R} \frac{d \theta}{d R}-Z_{1}^{2} \theta=0 & \left(R_{1} \leq R \leq R_{2}\right) \\ \frac{d^{2} \phi}{d R^{2}}+\frac{1}{R} \frac{d \phi}{d R}-Z_{2}^{2} \phi=0 & \left(R_{2} \leq R \leq 1\right)\end{cases}
$$

where

$$
R=r / r_{3} ; Z_{1}=Z_{0} / R_{1} ; Z_{2}=Z_{1} / \sqrt{\delta} ; Z_{0}=\sqrt{B i / \xi}
$$

The conservation of energy has to be satisfied at the section where step change occurred. Assuming negligible heat transfers through the tip, Eq. (2) is subjected to the following boundary conditions:

$$
\text { at } R= \begin{cases}R_{1}, & \theta=1 \\ R_{2}, & \theta=\phi \\ R_{2}, & R_{1} d \theta / d R+B i_{j}(1-\delta) \theta=\delta R_{1} d \phi / d R \\ 1, & d \phi / d R=0\end{cases}
$$

where, $\mathrm{Bi}_{\mathrm{j}}$ is the Biot number based on the convective heat transfer coefficient at the step surface, $h_{\mathrm{j}} r_{1} / k$ and $h_{\mathrm{j}}$ is the convective heat transfer coefficient at the step surface. To determine the temperature distribution, Eq. (2) is solved along with the boundary conditions (Eq. (4)). Basically, Eq. (2) is a modified Bessel equation, which was solved analytically as a function of modified Bessel functions [2]. Because of the associated infinite series function for the Bessel functions, calculations are difficult. Alternatively, this difficulty can be overcome by choosing an approximate analytical methodology. The approximate analytical techniques for this model are described in the following text.

Eq. (2) can be expressed as a function of a differential operator: 


$$
\begin{cases}\left(D^{2}+\frac{1}{R} D-Z_{1}^{2}\right) \theta=0 & \left(R_{1} \leq R \leq R_{2}\right) \\ \left(D^{2}+\frac{1}{R} D-Z_{2}^{2}\right) \varphi=0 & \left(R_{2} \leq R \leq 1\right)\end{cases}
$$

where operator $D=d / d R$. Here, $\mathrm{R}$ is a variable. An approximate solution is possible by choosing a constant $\mathrm{R}$ that can be determined using the mean value theorem along the length of the fin. Accordingly, the average value of the function $1 / R$ in a certain interval can be defined as

$$
\frac{1}{\bar{R}}=\int \frac{d R}{R} / \int d R
$$

On the two different intervals in Eq. (5), $\bar{R}$ is determined using Eq. (6),

$$
\bar{R}= \begin{cases}\left(R_{2}-R_{1}\right) / \ln \left(R_{2} / R_{1}\right) & \left(R_{1} \leq \bar{R} \leq R_{2}\right) \\ \left(1-R_{2}\right) / \ln \left(1 / R_{2}\right) & \left(R_{2} \leq \bar{R} \leq 1\right)\end{cases}
$$

Utilizing these average values, Eq. (5) yields

$$
\left\{\begin{array}{l}
\left(D^{2}+\frac{1}{\bar{R}} D-Z_{1}^{2}\right) \theta=0 \quad\left(R_{1} \leq \bar{R} \leq R_{2}\right) \\
\left(D^{2}+\frac{1}{\bar{R}} D-Z_{2}^{2}\right) \phi=0 \quad\left(R_{2} \leq \bar{R} \leq 1\right)
\end{array}\right.
$$

The roots of the auxiliary Eq. (8) are

$$
\left\{\begin{array}{l}
\left(D_{1}, D_{2}\right)=\left(-1 \pm \sqrt{1+4 Z_{1}^{2} \bar{R}^{2}}\right) / 2 \bar{R} \quad\left(R_{1} \leq \bar{R} \leq R_{2}\right) \\
\left(D_{3}, D_{4}\right)=\left(-1 \pm \sqrt{1+4 Z_{2}^{2} \bar{R}^{2}}\right) / 2 \bar{R} \quad\left(R_{2} \leq \bar{R} \leq 1\right)
\end{array}\right.
$$

The solution of Eq. (8) with boundary conditions Eq. (4) can be written as

$$
\begin{cases}\theta=\frac{\left(e^{D_{1} R+D_{2} R_{1}}-e^{D_{1} R_{1}+D_{2} R}\right) A+e^{D_{1} R_{2}+D_{2} R}-e^{D_{1} R+D_{2} R_{2}}}{e^{D_{1} R_{2}+D_{2} R_{1}}-e^{D_{1} R_{1}+D_{2} R_{2}}} & \left(R_{1} \leq \bar{R} \leq R_{2}\right) \\ \phi=\frac{\left(D_{4} e^{D_{3} R+D_{4}}-D_{3} e^{D_{3}+D_{4} R}\right) A}{\left(D_{4} e^{D_{3} R_{2}+D_{4}}-D_{3} e^{D_{3}+D_{4} R_{2}}\right)} & \left(R_{2} \leq \bar{R} \leq 1\right)\end{cases}
$$

where

$$
A=\frac{R_{1} \mu_{1} \mu_{5}}{R_{1} \mu_{2} \mu_{5}+B i_{j}(1-\delta) \mu_{3} \mu_{5}-R_{1} D_{3} D_{4} \mu_{3} \mu_{4}}
$$




$$
\left[\begin{array}{l}
\mu_{1} \\
\mu_{2} \\
\mu_{3} \\
\mu_{4} \\
\mu_{5}
\end{array}\right]=\left[\begin{array}{c}
D_{1} e^{D_{1} R_{2}+D_{2} R_{2}}-D_{2} e^{D_{1} R_{2}+D_{2} R_{2}} \\
D_{1} e^{D_{1} R_{2}+D_{2} R_{1}}-D_{2} e^{D_{1} R_{1}+D_{2} R_{2}} \\
e^{D_{1} R_{2}+D_{2} R_{1}}-e^{D_{1} R_{1}+D_{2} R_{2}} \\
e^{D_{3} R_{2}+D_{4}}-e^{D_{3}+D_{4} R_{2}} \\
D_{4} e^{D_{3} R_{2}+D_{4}}-D_{3} e^{D_{3}+D_{4} R_{2}}
\end{array}\right]
$$

The actual heat transfer rate $(q)$ in a fin is estimated by employing Fourier's law of heat conduction at the fin base and it is written in dimensionless form $(Q)$ as

$$
\begin{aligned}
Q & =\frac{q}{\pi k r_{1}\left(T_{b}-T_{a}\right)} \\
& =\frac{\xi R_{1}}{\mu_{3}}\left[D_{1} e^{D_{1} R_{1}+D_{2} R_{2}}-D_{2} e^{D_{1} R_{2}+D_{2} R_{1}}-A\left(D_{1} e^{D_{1} R_{1}+D_{2} R_{1}}-D_{2} e^{D_{1} R_{1}+D_{2} R_{1}}\right)\right]
\end{aligned}
$$

The actual heat transfer $\operatorname{rate}(q)$ can also be possible to calculate by using the Newton law of cooling for the integration over the entire fin surface,

$$
\begin{aligned}
& Q=\frac{q}{\pi k r_{1}\left(T_{b}-T_{a}\right)} \\
& =\frac{B i \mu_{3}^{-1}}{R_{1}^{2} D_{1}^{2} D_{2}^{2}}\left[A D_{2}^{2}\left(D_{1} R_{2}-1\right) e^{D_{1} R_{2}+D_{2} R_{1}}-A D_{2}^{2}\left(D_{1} R_{1}-1\right) e^{D_{1} R_{1}+D_{2} R_{1}}\right. \\
& \quad-A D_{1}^{2}\left(D_{2} R_{2}-1\right) e^{D_{1} R_{1}+D_{2} R_{2}}+A D_{1}^{2}\left(D_{2} R_{2}-1\right) e^{D_{1} R_{1}+D_{2} R_{1}} \\
& \quad+D_{1}^{2}\left(D_{2} R_{2}-1\right) e^{D_{1} R_{2}+D_{2} R_{2}}-D_{1}^{2}\left(D_{2} R_{2}-1\right) e^{D_{1} R_{1}+D_{2} R_{2}} \\
& \left.\quad-D_{2}^{2}\left(D_{1} R_{2}-1\right) e^{D_{1} R_{2}+D_{2} R_{2}}+D_{2}^{2}\left(D_{1} R_{1}-1\right) e^{D_{1} R_{1}+D_{2} R_{2}}\right] \\
& \quad+\frac{B i \mu_{5}^{-1}}{R_{1}^{2} D_{3}^{2} D_{4}^{2}}\left[D_{4}^{3}\left(D_{3}-1\right) e^{D_{3}+D_{4}}-D_{4}^{3}\left(D_{3} R_{2}-1\right) e^{D_{3} R_{2}+D_{4}}\right. \\
& \left.\quad-D_{3}^{3}\left(D_{4}-1\right) e^{D_{3}+D_{4}}+D_{3}^{3}\left(D_{4} R_{2}-1\right) e^{D_{3}+D_{4} R_{2}}\right]+\frac{B i}{R_{1}}(1-\delta) R_{2} A \xi
\end{aligned}
$$

The ideal heat transfer rate $\left(q_{i}\right)$ is determined from the following dimensionless expression,

$$
Q_{i}=\frac{q_{i}}{4 \pi r_{1} k\left(T_{b}-T_{a}\right)}=\frac{B i}{2 R_{1}^{2}}\left[1-R_{1}^{2}+\xi R_{1} R_{2}(1-\delta) B i_{j} / B i\right]
$$

The expression for fin effectiveness can be expressed by

$$
\varepsilon=\frac{\eta\left[1-R_{1}^{2}+\xi(1-\delta) R_{1} R_{2} B i_{j} / B i\right]}{2 R_{1}^{2} \xi}
$$

After knowing the actual and ideal heat transfer rates, the fin efficiency $(\eta)$ and the fin effectiveness $(\varepsilon)$ can readily be obtained. Here it can be noted that the integral and differential approaches for the fin heat transfer provides the same result for an exact analytical solution. However, from the perspective of an approximate analytical analysis, the integral approach may yield a better prediction of the heat transfer rate. 


\section{Results and discussion}

From the analysis, it can be noted that the temperature distribution in the fin is a function of five design parameters, $Z_{0}, \delta, R_{1}, R_{2}$, and $B i_{j}$. Fig. 2 is drawn for the effects of $Z_{0}$ on the temperature distribution. An increase in $Z_{0}$ decreases the temperature sharply. Fig. 2 shows an important aspect for predicting the temperature for an annular step fin by the approximate method, which is exactly the same as when it is determined using the exact method. It can be highlighted that the designer can adopt the approximate method for temperature distribution in an annular step fin without compromising any accuracy level of results.

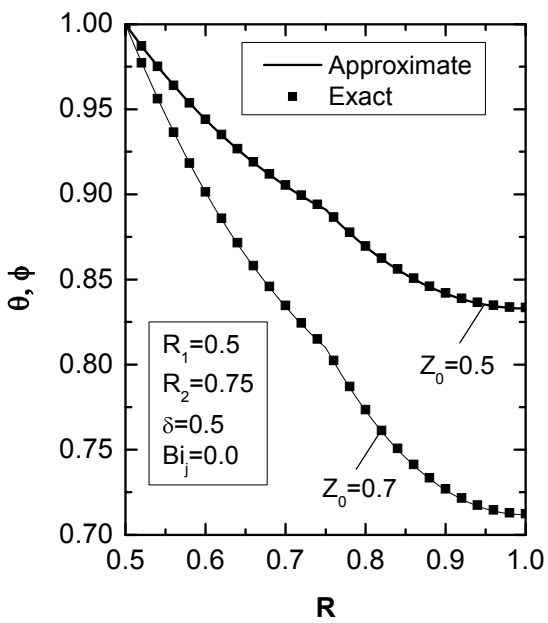

Figure 2: Comparison of temperature distribution by exact and approximate analytical techniques.

Fig. 3 depicts the fin efficiency and fin effectiveness as a function of the fin parameter $\mathrm{Z}_{0}$ for different values of $\delta$. Clearly, at lower values of $\mathrm{Z}_{0}$, the fin performance is high, with a weak function of $\delta$. From the figure, it is noted that the fin efficiency and fin effectiveness values calculated using the exact and approximate methods differ slightly. Nevertheless, the agreement between these results is reasonably acceptable. On the other hand, the integral approach (int.) for the determination of the heat transfer rate is more accurate than that determined by the differential approach (diff.) for the approximate analysis, whereas, the exact analysis provides unaltered results.

To get an actual idea about the accuracy of the referred approximate analytic technique, a study has been carried out. In this case, a table has been prepared to compare fin performance from both the exact and the approximate methods. The efficiency calculation by exact and approximate means has been presented in Table 1. It shows the percentage error in the measurement of fin efficiency with different values of fin parameter $\mathrm{Z}_{0}$. An insignificant error has been found for 
predicting the temperature and the fin efficiency. The error in fin efficiency is dependent not only on $Z_{0}$ but also on the method of calculating heat transfer rate by either integral or differential approaches. Irrespective of the design condition for fin efficiency, the integral approach always shows less error compared to the differential approach. Again, an increasing error is found with an increase in $Z_{0}$.

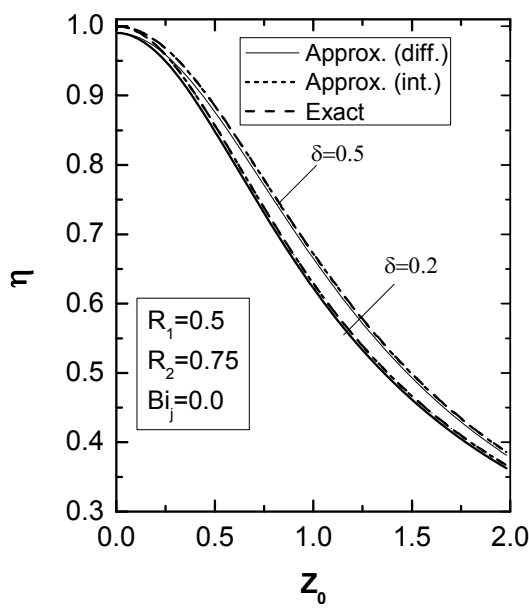

(a) Fin efficiency

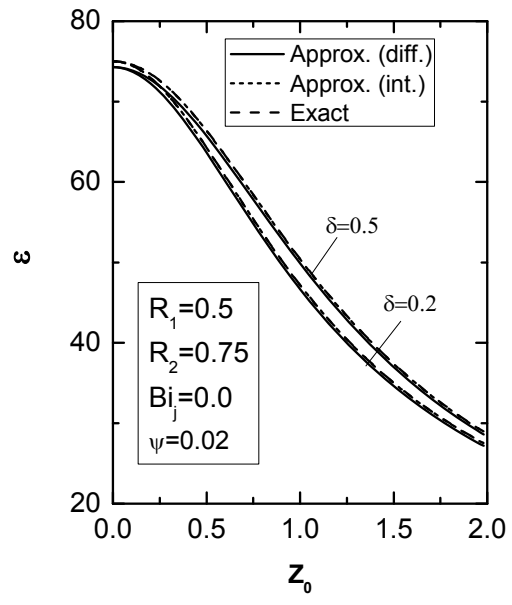

(b) Fin effectiveness

Figure 3: A comparison of prediction of results for fin performances with fin parameter $\mathrm{Z}_{0}$ and fin thickness ratio $\delta$.

Table 1: Fin efficiency at a design condition $\left(R_{1}=0.5, R_{2}=0.75, \delta=0.5\right.$, and $B i_{j}=0.0$ ).

\begin{tabular}{cccccc}
\hline$Z_{0}$ & \multicolumn{5}{c}{ Efficiency } \\
\cline { 2 - 6 } & $\begin{array}{c}\text { Exact } \\
\eta\end{array}$ & $\begin{array}{c}\text { Approx. } \\
\eta_{\text {dif }}\end{array}$ & $\begin{array}{c}\text { Error } \\
\%\end{array}$ & $\begin{array}{c}\text { Approx. } \\
\eta_{\text {int }}\end{array}$ & $\begin{array}{c}\text { Error } \\
\%\end{array}$ \\
\hline 0.1 & 0.9947 & 0.9852 & 0.963 & 0.9947 & 0.0 \\
0.2 & 0.9793 & 0.9698 & 0.971 & 0.9793 & 0.0 \\
0.3 & 0.9549 & 0.9455 & 0.983 & 0.9547 & 0.016 \\
0.4 & 0.9229 & 0.9137 & 0.998 & 0.9227 & 0.028 \\
0.5 & 0.8854 & 0.8764 & 1.016 & 0.8850 & 0.040 \\
0.6 & 0.8441 & 0.8354 & 1.036 & 0.8437 & 0.054 \\
0.7 & 0.8009 & 0.7924 & 1.056 & 0.8004 & 0.067 \\
0.8 & 0.7572 & 0.7490 & 1.076 & 0.7566 & 0.079 \\
0.9 & 0.7141 & 0.7063 & 1.096 & 0.7135 & 0.090 \\
1.0 & 0.6725 & 0.6650 & 1.114 & 0.6718 & 0.099 \\
\hline
\end{tabular}




\section{Conclusions}

In this study, an approximate analytical effort has been developed to determine the temperature distribution and performance of a single annular step fin under convective environment conditions. Based on the previous discussion of results, the following conclusions can be summarized as follows:

1. The temperature distribution from the approximate method is exactly the same with the exact analytical value. The accuracy level is also dependent on the fin parameter $Z_{0}$.

2. The fin heat transfer by the approximate method is dependent on the method of determination. Newton's law yielded an accurate prediction compared to that determined by Fourier's law. However, both approaches show a high accuracy.

3. Finally, the approximate method developed in the present study for annular step fins shows high accuracy. Since the approximate analysis is so simple, hand calculation is possible to avoid the rigorous calculation required in the case of exact analysis.

\section{References}

[1] Kundu, B., Bhanja, D. \& Lee, K.-S., A model on the basis of analytics for computing maximum heat transfer in porous fins. Int. J. Heat Mass Trans., 55, pp. 7611-7622, 2012.

[2] Kundu B. \& Das P.K., Performance analysis and optimization of annular fin with a step change in thickness. ASME J. Heat Trans. 123, pp. 601-604, 2001 .

[3] Kraus, A.D., Aziz, A. \& Welty, J.R., Extended surface heat transfer. John Wiley, NY, 2001.

[4] Ullman, A. \& Kalman, H., Efficiency and optimized dimensions of annular fins of different cross section shapes. Int. J. Heat Mass Trans. 32, pp. 11051110, 1989.

[5] Kundu, B., Performance and optimization analysis of SRC profile fins subject to simultaneous heat and mass transfer. Int. J. Heat Mass Trans. 50, pp. 1545-1558, 2007.

[6] Arslanturk, C., Simple correlation equations for optimum design of annular fins with uniform thickness. Appl. Thermal Engng. 25, pp. 2463-2468, 2005.

[7] Aziz, A. \& Fang, T., Alternative solutions for longitudinal fins of rectangular, trapezoidal and concave parabolic profiles. Energy Convers. Manage. 51, pp. 2188-2194, 2010.

[8] Kundu, B., Analysis of thermal performance and optimization of concentric circular fins under dehumidifying conditions. Int. J. Heat Mass Trans. 52, pp. 2646-2659, 2009.

[9] Kundu, B. \& Das, P.K., Performance analysis and optimization of eccentric annular disk fins. ASME J. Heat Transfer. 121(1), pp. 128-135, 1999. 
[10] Sharqawy, M.H. \& Zubair, S.M., Efficiency and optimization of an annular fin with combined heat and mass transfer- an analytical solution. Int. J. Refrig. 30, pp. 751-758, 2007.

[11] Rosario, L. \& Rahman, M.M., Overall efficiency of a radial fin assembly under dehumidifying conditions. ASME J. Energy Resour. Technol. 120, pp. 299-305, 1998.

[12] Almogbel, M. \& Bejan, A., Cylindrical trees of pin fins. Int. J. Heat Mass Trans., 43(23), pp. 4285-4297, 2000.

[13] Kundu, B. Bhanja, D., Performance and optimization analysis of a constructal T-shaped fin subject to variable thermal conductivity and convective heat transfer coefficient. Int. J. Heat Mass Trans., 53, pp. 254 267, 2010.

[14] Kundu, B. \& Bhanja, D., Thermal analysis of a constructal T-shaped porous fin with radiation effects. Int. J. Refrig., 34(6), pp. 1483-1496, 2011.

[15] Kundu, B. \& Bhanja, D., Radiation effect on optimum design analysis of a constructal T-shaped fin with variable thermal conductivity. Heat Mass Transfer, 48(1), pp. 109-122, 2012. 